Check for updates

Cite this: RSC Adv., 2019, 9, 10072

\title{
Efficient synthesis of epoxybutane from butanediol via a two-step process $\uparrow$
}

\author{
Xin Niu, ${ }^{\text {ab }}$ Liguo Wang, (D) *bcd Junya Cao, ${ }^{a}$ Yan Cao, ${ }^{\text {b }}$ Peng He, ${ }^{\text {b }}$ Junya Zhou ${ }^{\text {ab }}$ \\ and Huiquan $\mathrm{Li}$ (iD) *bc
}

A novel approach for the synthesis of epoxybutane via decarboxylation of butenyl carbonate derived from butanediol was developed for the first time. For the carbonylation of butanediol with dimethyl carbonate, $\mathrm{NaAlO}_{2}$ has exhibited excellent catalytic activity under mild reaction conditions. The yield of butenyl carbonate reached as high as $96.2 \%$. $\mathrm{NaAlO}_{2}$ provides suitable acid-base active sites to promote the transesterification reaction of butanediol and dimethyl carbonate. For the following step of decarboxylation of butenyl carbonate, ionic liquid 1-butyl-3-methylimidazolium bromide could effectively catalyze the decarboxylation process both in batch or continuous processes. Moreover, the catalytic mechanism for the crucial step of decarboxylation of butenyl carbonate over 1-butyl-3methylimidazolium bromide was explored using DFT calculations. The results showed that the electrostatic and hydrogen-bond effects of 1-butyl-3-methylimidazolium bromide played a crucial role for the generation of epoxybutane. Briefly, the $\mathrm{Br}$ anion of the ionic liquid attacks the methylene of the ring and the $\mathrm{H}$ of the ionic liquid cation attacks the carbonyl oxygen, which facilitated the five-ring opening and subsequent decarboxylation to form BO. This study not only provided a new and green synthetic route for producing epoxybutane, but also contributed to the effective utilization of butanediol, which is inevitably produced as by-product in the process of coal to ethylene glycol, suggesting a promising application in the clean manufacture of epoxybutane with inexpensive cost.

Received 17th February 2019

Accepted 25th March 2019

DOI: $10.1039 / c 9 r a 01220 f$

rsc.li/rsc-advances

\section{Introduction}

Epoxybutane (BO) is an important organic chemical raw material and mainly used to synthesize polyether polyol, ${ }^{1}$ which is being used in the manufacture of value-added polyurethane. ${ }^{2}$ Until now, the monomer of polyether polyol is mainly chosen from ethylene oxide (EO), propylene oxide (PO) and $\mathrm{BO}^{3,4}$ Compared with EO and PO, BO not only possess a similar activity that can react with hydrogen containing compounds, such as water, alcohol, phenols, sulfur alcohol, ammine, acids and so on, but also has unique properties that can improve the durability of diphenylmethane diisocyanate (MDI)-based polyurethane. However, owing to the restrictions from the severe safety issues in the production technology and the expensive cost, the market share of polyether polyol derived from BO is

${ }^{a}$ China University of Mining \&Technology, Beijing, Beijing, 100083, P. R. China

${ }^{b}$ CAS Key Laboratory of Green Process and Engineering, National Engineering Laboratory for Hydrometallurgical Cleaner Production Technology, Institute of Process Engineering, Chinese Academy of Sciences, Beijing, 100190, P. R. China. E-mail: lgwang@ipe.ac.cn; hqli@ipe.ac.cn

${ }^{c}$ Sino-Danish College University, Chinese Academy of Sciences, Beijing, 100049, P. R. China

${ }^{d}$ Dalian National Laboratory for Clean Energy, Dalian, 116023, China

$\dagger$ Electronic supplementary information (ESI) available. See DOI: 10.1039/c9ra01220f smaller than that made from $\mathrm{EO}$ and PO. At present, $\mathrm{BO}$ is being synthesized by chlorohydrin process, direct oxidation method and indirect oxidation method. ${ }^{5}$ These conventional manufacturing processes of BO suffer from several drawbacks, such as environmental pollution, harsh operation conditions, and high cost of the raw materials. In the chlorohydrin route, butylene is reacted with water and $\mathrm{Cl}_{2}$ to produce chlorobutanol, which is then reacted with base to generate BO. In the production of $\mathrm{BO}$ by chlorohydrin process, salt waste and large amount of wastewater will be produced. In addition, the production of large amounts of hydrochloric acid will corrode the equipment. In the indirect oxidation method, the styrene process is the main process. Ethyl benzene is first oxidized with $\mathrm{O}_{2}$, where the peroxy compound is used to generate $\mathrm{BO}$ from butylene. The residual aromatic alcohol is then dehydrated to styrene. This method is energy-intensive and can produce sizeable waste streams. The direct oxidation method is the oxidation of butene to $\mathrm{BO}$ by hydrogen peroxide. ${ }^{6}$ Its disadvantages are harsh operation conditions and high costs of the raw materials. In this context, a new and environmentally friendly route for the synthesis of BO from butanediol via butenyl carbonate (BC) intermediate was investigated in this paper. The new route includes the synthesis of $\mathrm{BC}$ from butanediol and DMC, followed by decarboxylation of $\mathrm{BC}$ to $\mathrm{BO}$, as shown in Scheme 1. 
(1)

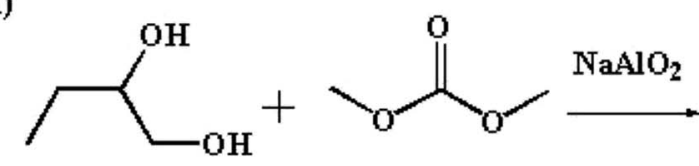

1,2-Butanediol

Dim ethyl c arbonate

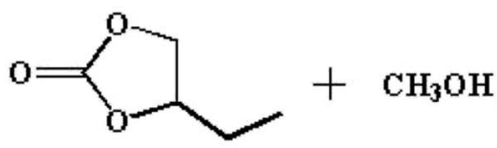

1,2-Butylenecarb onate methanol

(2)

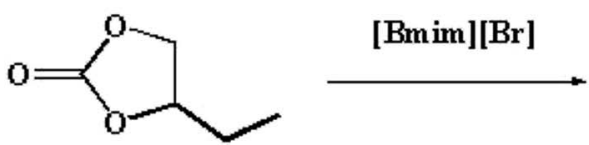

1,2-Butylenecarbonate

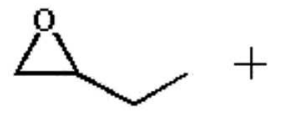

1,2-ep oxybutane
$\mathrm{O}=\mathrm{C}=\mathrm{O}$

carbon dioxide

Scheme 1 (1) Synthesis of BC from butanediol and DMC, (2) BC decarboxylation to BO.

In the previous reports, the intermediate of $\mathrm{BC}$ is generally synthesized from $\mathrm{BO}$ and $\mathrm{CO}_{2}$, which have the disadvantages of relatively high pressure and high cost. ${ }^{7-12}$ The development of a new route for the effective synthesis of $\mathrm{BC}$ via an inexpensive route is highly desired. In terms of this reason, a new approach based on the transesterification of butanediol with dimethyl carbonate (DMC) was developed in our group, where the crucial raw material of butanediol could be readily obtained from the by-product, which is inevitably produced in the well-established process of coal to ethylene glycol process. ${ }^{13}$ Owing to the high activity of alcohol hydroxyl group, ethylene glycol can be further hydrogenated to ethanol in the presence of hydrogen. Then the ethylene glycol can reacts with ethanol to produce butanediol due to the Golbert reaction. ${ }^{14,15}$ Thus, inexpensive butanediol is produced in large quantities in the coal to ethylene glycol process.

The development of effective catalyst system is crucial for the synthesis of $\mathrm{BC}$ from butanediol and DMC. Sodium aluminate $\left(\mathrm{NaAlO}_{2}\right)$ is generally used as a cheaply available and highly basic catalyst. ${ }^{16-22}$ As it is insoluble in many organic solvents, $\mathrm{NaAlO}_{2}$ can be used as a truly heterogeneous catalyst. ${ }^{23,24}$ Some researchers reported that $\mathrm{NaAlO}_{2}$ have been used as an active catalyst for a number of base-catalyzed reactions (transesterification, isomerization, ${ }^{25,26}$ and condensation). Therefore, owing to its unique features in basicity, $\mathrm{NaAlO}_{2}$ was also employed in the synthesis of $\mathrm{BC}$ via carbonylation of butanediol with DMC. In addition, the synthesis of BO from decarboxylation of $\mathrm{BC}$ is worthy of special attention in terms of economic and environmental points of view. Imidazolium-based ionic liquid was demonstrated to be capable of catalyzing the decarboxylation of glycerol carbonate. ${ }^{27-32}$ In this work, imidazoliumbased ionic liquids were tested and applied as catalyst in the decarboxylation of $\mathrm{BC}$ to produce $\mathrm{BO}$.

In this paper, a novel approach for the synthesis of epoxybutane (BO) via decarboxylation of butenyl carbonate (BC) derived from butanediol were successfully developed. In this process, $\mathrm{NaAlO}_{2}$ was used as catalyst in the transesterification of butanediol with dimethyl carbonate (DMC) to produce $\mathrm{BC}$, and subsequently 1-butyl-3-methylimidazolium bromide ([Bmim] [Br]) was used as catalyst in the decarboxylation of $\mathrm{BC}$ to produce $\mathrm{BO}$. The influence of reaction parameters, e.g. reaction temperature, reaction time and catalyst amount on the catalytic performance were optimized. Furthermore, the crucial catalytic mechanism of decarboxylation of $\mathrm{BC}$ to $\mathrm{BO}$ is deeply explored using DFT calculations.

\section{Experimental}

\section{Materials}

Dimethyl carbonate (99\%) and decane were purchased from Aladdin Industrial Corporation. Butanediol (98\%), 1,2-epoxybutane and butylene carbonate $(98 \%)$ were purchased from Tokyo Chemical Industry Co., Ltd. Sodium aluminate and zinc bromide were purchased from Sinopharm Chemical Reagent Co., Ltd. 1-Butyl-3-methylimidazolium bromide ([Bmim][Br]), 1butyl-3-methylimidazolium chloride ([Bmim][Cl]), 1-butyl-3methylimidazolium iodide ([Bmim][I]) and 1-butyl-3methylimidazolium tetrafluoroborate $\left([\mathrm{Bmim}]\left[\mathrm{BF}_{4}\right]\right)$ were purchased from Shanghai Cheng Jie Chemical Co. LTD.

\section{Catalytic activity test}

Carbonylation of butanediol with dimethyl carbonate. Carbonylation reaction between butanediol and DMC was carried out in a $100 \mathrm{~mL}$ autoclave with a magnetic stirring. In a typical procedure, $22.4 \mathrm{~g}$ dimethyl carbonate and $4.5 \mathrm{~g}$ butanediol was loaded to the reactor, followed by the addition of $10 \mathrm{wt} \%$ catalyst $(0.45 \mathrm{~g})$. The mixture was heated to $120^{\circ} \mathrm{C}$ with magnetic stirring and condenser, and kept for a certain time, and then cooled down to the room temperature. After the reaction, the catalyst was separated by centrifugation, and then washed by DMC and reused again.

Decarboxylation of BC. Decarboxylation of BC was tested by both batch and continuous processes. In batch cases, BC was loaded to the $250 \mathrm{~mL}$ three-necked flask equipped with condensers and an oil bath, and was decarboxylated into BO in the presence of catalyst at a specified temperature under the vacuum degree of $0.005 \mathrm{MPa}$. BO produced during the decarboxylation reaction was collected through a three-stage condensing device. 
While for continuous process, BC was loaded to the $250 \mathrm{~mL}$ three-necked flask from the sample bottle which was put on the balance. Thus, the amount of the BC introduced into the reactor can be directly calculated. Then $\mathrm{BC}$ was continuous fed into the flask by vacuum aspiration, and the feeding rate of $\mathrm{BC}$ was controlled by the valve under the vacuum degree of $0.005 \mathrm{MPa}$. During the reaction, the product was continuously collected in the bottom of flask and weighted. As a result, the yield of BO can be calculated. ${ }^{6}$ The device for the continuous process is shown in Fig. S1. $\dagger$

Two-step process for the synthesis of BO by using BC from butanediol. In two-step process, firstly, the synthesis of BC was carried out in a $1 \mathrm{~L}$ reaction autoclave under the optimal conditions. Then BC was purified by the distillation process, and was consequently charged to the decarboxylation reactor, and converted to $\mathrm{BO}$ through the continuous device.

\section{Product analysis}

After the reaction, the reaction mixture was analyzed using a gas chromatograph (GC-2010) using a RTX-WAX (30 m $\times 0.25 \mathrm{~mm}$ $\times 0.25 \mu \mathrm{m})$ capillary column and a flame ionization detector (FID). The decane was used as an internal standard for the quantitative analysis of butanediol, BC and BO. BC conversion, $\mathrm{BC}$ yield, BC selectivity, $\mathrm{BO}$ yield and formation rate of the decarboxylation of butenyl carbonate were calculated using the following equations:

$$
\begin{gathered}
C_{\text {butanediol }}=\frac{M_{\mathrm{rt} 0}-M_{\mathrm{rtn}}}{M_{\mathrm{rt} 0}} \times 100 \% \\
Y_{\mathrm{BC}}=\frac{M_{\mathrm{itn}}}{M_{\mathrm{rt} 0}} \times 100 \% \\
S_{\mathrm{BC}}=\frac{M_{\mathrm{itn}}}{M_{\mathrm{rt} 0}-M_{\mathrm{rtn}}} \times 100 \% \\
Y_{\mathrm{BO}}=\frac{M_{\mathrm{BO}}}{M_{\mathrm{BC}}} \times 100 \% \\
\mathrm{FR}_{\mathrm{BO}}=\frac{M_{\mathrm{BO}}}{M_{\text {catalyst }} h} \times 100 \%
\end{gathered}
$$

where $C_{\text {butanediol }}$ is the conversion of butanediol; $Y_{\mathrm{BC}}$ is the yield of BC; $S_{\mathrm{BC}}$ is the selectivity of BC; $M_{\mathrm{rto}}$ is the initial mole amount of butanediol; $M_{\mathrm{rtn}}$ is the mole amount of butanediol; $M_{\mathrm{itn}}$ is the mole amount of $\mathrm{BC}$ produced; $Y_{\mathrm{BO}}$ is the yield of $\mathrm{BO} ; M_{\mathrm{BO}}$ is the mole amount of $\mathrm{BO}$ produced; $M_{\mathrm{BC}}$ is the initial mole amount of $\mathrm{BC} ; \mathrm{FR}_{\mathrm{BO}}$ is the formation rate of the decarboxylation of butenyl carbonate; $M_{\text {catalyst }}$ is the mole amount of catalyst; $h$ is the reaction time of decarboxylation.

\section{Characterization}

XRD: phase structure of catalyst was determined with X-ray diffractometer using $\mathrm{Cu} \mathrm{K} \alpha$ radiation. The working voltage and current were $40 \mathrm{kV}$ and $40 \mathrm{~mA}$, respectively. The $2 \theta$ range used was from $5^{\circ}$ to $90^{\circ}$.

Infrared (IR) analysis of the catalyst samples (diluted by $\mathrm{KBr}$ at a ratio of $1: 200$ ) were characterized using a TENSOR-27 spectrometer (Bruker) with the scanning range is 400$4000 \mathrm{~cm}^{-1}$.

${ }^{1} \mathrm{H}$ NMR spectra were recorded on a Bruker Avance III 600 $\mathrm{MHz}$ spectrometer in $\mathrm{CDCl}_{3}$ with tetramethylsilane (TMS) as internal standard.

$\mathrm{CO}_{2}$-TPD was carried out to determine the basicity of samples using an AutoChem II 2920 equipped with a thermal conductivity detector (TCD). About $100 \mathrm{mg}$ of sample was heated in situ in $25 \mathrm{~mL} \min ^{-1}$ of helium gas at $220^{\circ} \mathrm{C}$ for $0.5 \mathrm{~h}$ in order to remove adsorbed impurities. After that, the sample was cooled to $50{ }^{\circ} \mathrm{C}$ and saturated with $25 \mathrm{~mL} \mathrm{~min}^{-1}$ of $10 \%$ vol\% $\mathrm{CO}_{2} / \mathrm{He}$ for $1 \mathrm{~h}$. Weakly adsorbed $\mathrm{CO}_{2}$ was eliminated by flushing with a $25 \mathrm{~mL} \mathrm{~min}{ }^{-1}$ of helium gas at $50{ }^{\circ} \mathrm{C}$ for $0.5 \mathrm{~h}$, and increased to $800{ }^{\circ} \mathrm{C}$ with a ramping rate of $10{ }^{\circ} \mathrm{C} \mathrm{min} \mathrm{m}^{-1}$

$\mathrm{N}_{2}$ sorption measurement was performed using a Quantachrome Autosorb- 1 at $77 \mathrm{~K}$ and the samples were degassed under vacuum at $573 \mathrm{~K}$ for $3 \mathrm{~h}$ before measurement. The specific surface areas were calculated by the Brunauer-EmmettTeller (BET) method using $\mathrm{N}_{2}$ adsorption data ranging from $P / P_{0}$ $=0.05$ to 0.30 .

\section{Computational calculations}

All the Density Functional Theory (DFT) calculations were performed using the Dmol3 program available in Materials studio 6.1 package. The generalized gradient approximation (GGA) with Perdew-Wang 1991 function was used..334 Geometry optimizations for minima were carried out using the standard Berny algorithm in redundant internal coordinates up to the neighborhood of the solution. If not converged, the optimization was further continued using analytical second derivatives. Optimizations for transition states were carried out with an initial guess for the transition state being generated from manual manipulation of the geometry using MOLDEN. Complete linear synchronous transit and quadratic synchronous transit (LST/QST) calculations were performed to obtain the structures of transition states (TS)..$^{35,36}$

\section{Results and discussion}

\section{Transesterification of butanediol with DMC to synthesize BC}

Effect of reaction conditions on the BC synthesis. A proper catalyst with unique basicity is very important for obtaining effective catalytic performance in the transesterification reaction. In this work, sodium aluminate $\left(\mathrm{NaAlO}_{2}\right)$ were used as the catalyst for $\mathrm{BC}$ synthesis. $\mathrm{NaAlO}_{2}$ provides a suitable acid-base active site to promote the transesterification reaction of butanediol and DMC. The acid-base active sites of $\mathrm{NaAlO}_{2}$ is detected by $\mathrm{CO}_{2}$-TPD and $\mathrm{NH}_{3}$-TPD, and the results is shown in Fig. S2. $\dagger$ From Fig. S2, $\dagger$ it can be observed that the desorption peaks at $256{ }^{\circ} \mathrm{C}$ of $\mathrm{NaAlO}_{2}$ are attributed to the interaction of $\mathrm{CO}_{2}$ with weak basic sites. Meanwhile, the desorption peaks at $160{ }^{\circ} \mathrm{C}$ and $500{ }^{\circ} \mathrm{C}$ are attributed to the interaction of $\mathrm{NH}_{3}$ with 
acid sites of $\mathrm{NaAlO}_{2}$, which possibly originated from Lewis acid site of $\mathrm{Al}^{3+}$. It means that $\mathrm{NaAlO}_{2}$ contains both acid and base active sites. In the transesterification reaction, acid site accepts electrons as electron acceptors, while base site gives electrons as electron donors. They activate transesterification reaction substrates synergistically.

The effects of reaction conditions, such as catalyst amount, reaction temperature, DMC/butanediol molar ratio, as well as reaction time on the transesterification performance were investigated and discussed below.

The effect of catalyst amount ranging from 0.1-5.0 wt $\%$ (based on the butanediol amount) on the reaction was investigated in Fig. 1. It is observed that, without catalyst, the reaction efficiency is so low that, the butanediol conversion is only $6.7 \%$. However, the introduction of $0.1 \mathrm{~mol} \%$ catalyst led to the rapid increase of butanediol conversion to $95.5 \%$, then hardly changed when continue increasing the catalyst amount to $3.0 \mathrm{wt} \%$. Thus, the $0.1 \mathrm{~mol} \%$ catalyst was good enough for the transesterification of butanediol with DMC.

Fig. 2 showed the influence of temperature on the $\mathrm{BC}$ synthesis with $\mathrm{NaAlO}_{2}$ as catalyst. It can be observed that the BC is effectively synthesized with excellent selectivity within the temperature range investigated. Meanwhile, the butanediol conversion increased sharply from $52.2 \%$ to $94.8 \%$ at first with the temperature increased from 50 to $105{ }^{\circ} \mathrm{C}$. When the temperature increased to higher temperatures, i.e. $120{ }^{\circ} \mathrm{C}$ and $150{ }^{\circ} \mathrm{C}$, butanediol conversion gradually increased to $96.9 \%$. The above results indicated that temperature had a great effect on butanediol conversion, and the conversion rate became very fast and nearly completely converted at $120^{\circ} \mathrm{C}$.

The effect of molar ratio of DMC and butanediol was studied, as shown in Fig. 3. The conversion of butanediol increased from $91.5 \%$ to $96.6 \%$ with increasing the molar ratio from 2 to 4 , and further increase the molar ratio did not show any change. Thus DMC/butanediol molar ratio of $4: 1$ was chosen as the prefered

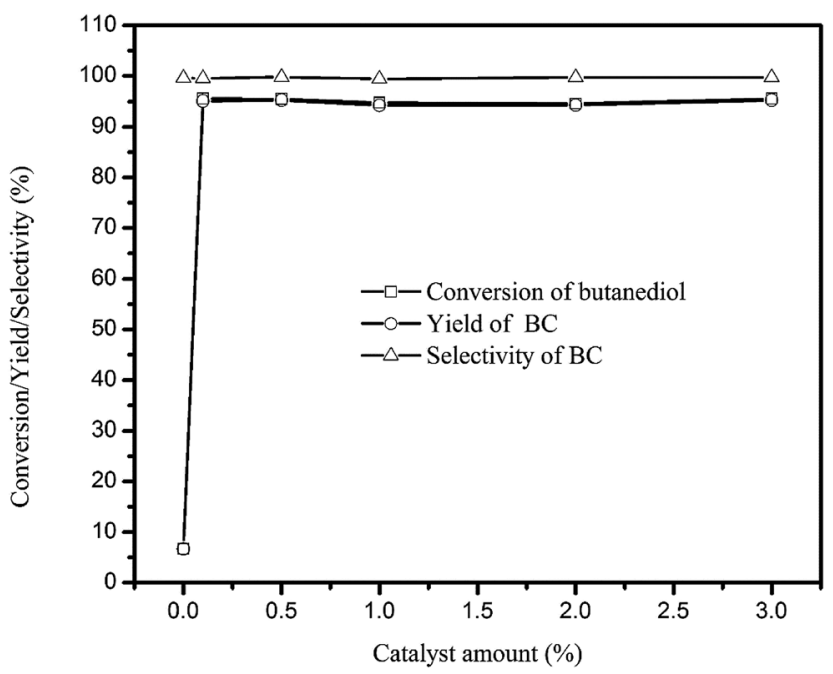

Fig. 1 Effect of catalyst amount on $\mathrm{BC}$ synthesis. (Reaction conditions: butanediol: $0.05 \mathrm{~mol} ; n(\mathrm{DMC}) / \mathrm{n}$ (butanediol) $=4$; temperature: $120^{\circ} \mathrm{C}$; reaction time: $60 \mathrm{~min}$.)

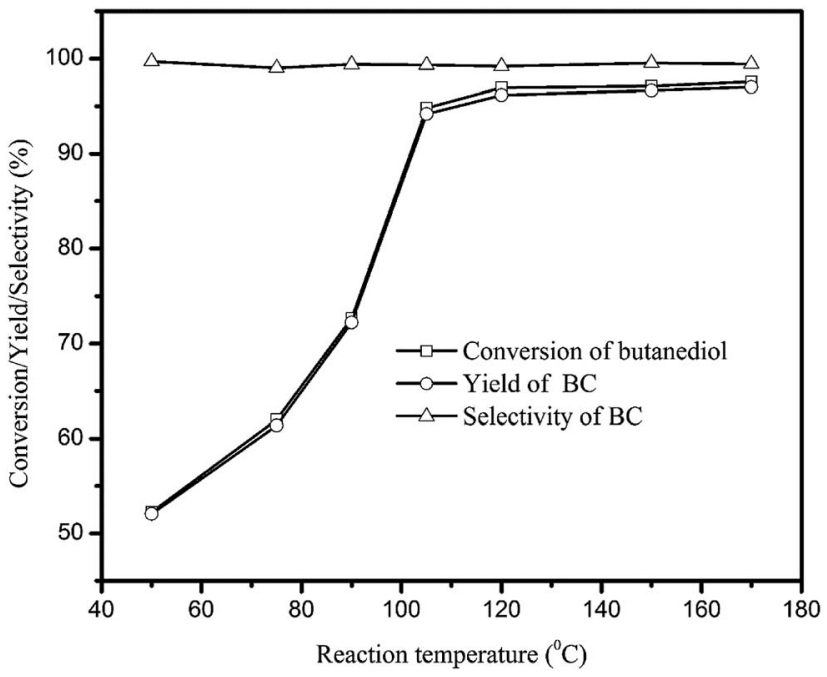

Fig. 2 Effect of temperature on $\mathrm{BC}$ synthesis. (Reaction conditions: butanediol: $0.05 \mathrm{~mol} ; n(\mathrm{DMC}) / \mathrm{n}$ (butanediol) $=5$; catalyst: $10 \mathrm{wt} \%$ of $\mathrm{NaAlO}_{2}$ based on butanediol; reaction time: $60 \mathrm{~min}$.)

value, in which case butanediol was completely converted into the decarboxylation precursor of BC.

At last, the effect of reaction time was also studied, and the result is shown in Fig. 4 . As can be seen from the figure, the reaction is very fast that the butanediol conversion reached to $78.9 \%$ only after $15 \mathrm{~min}$, and then quickly reached to $93.8 \%$ and kept unchanged anymore at $30 \mathrm{~min}$, which means $30 \mathrm{~min}$ is the equilibrium and optimum time for this reaction.

\section{Recyclability of the catalysts}

Additionally, the recyclability of the catalyst was also studied. After the reaction, the catalyst was separated by centrifugation,

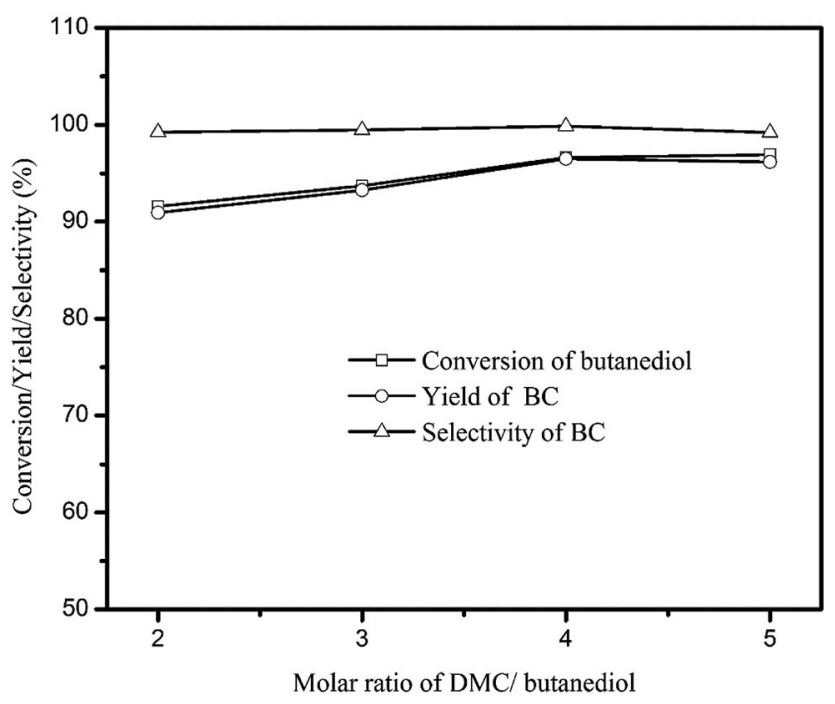

Fig. 3 Effect of molar ratio between DMC and butanediol on BC synthesis. (Reaction conditions: butanediol: 0.05 mol; catalyst: $10 \mathrm{wt} \%$ of $\mathrm{NaAlO}_{2}$ based on butanediol; temperature: $120{ }^{\circ} \mathrm{C}$; reaction time: 60 min.) 


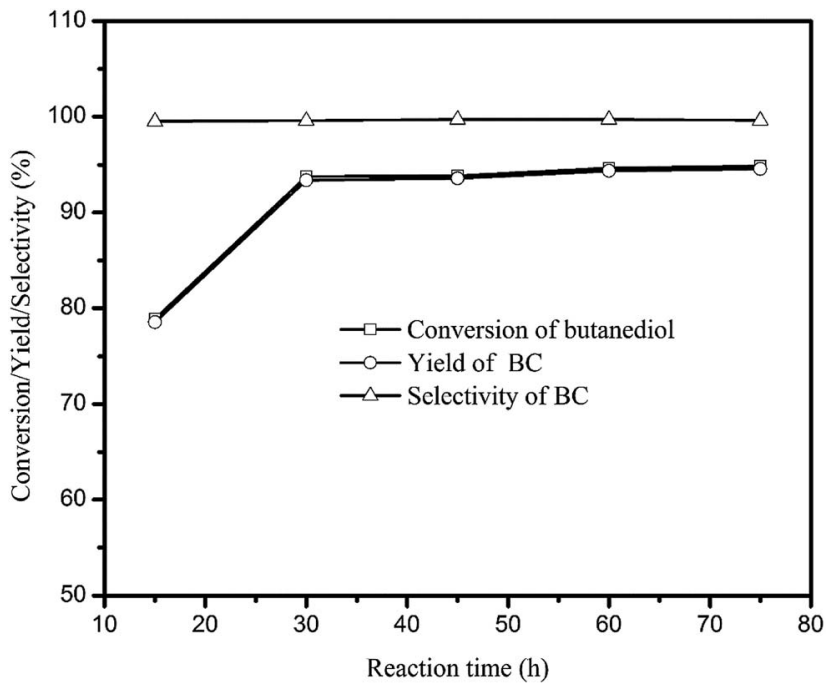

Fig. 4 Effect of reaction time on $\mathrm{BC}$ synthesis. (Reaction conditions: butanediol: $0.05 \mathrm{~mol} ; n(\mathrm{DMC}) / \mathrm{n}$ (butanediol) $=4$; catalyst: $10 \mathrm{wt} \%$ of $\mathrm{NaAlO}_{2}$ based on butanediol; temperature: $120{ }^{\circ} \mathrm{C}$.)

then washed by DMC and reused again. The result was shown in Fig. 5, it can be seen that, although BC selectivity was almost remained at $99.7 \%$, the butanediol conversion increased from $92.2 \%$ to $96.8 \%$ when fresh and reused catalyst were applied, respectively. From this result, it can be deduced that the catalytic activity was further enhanced during the first run, and exhibiting higher catalytic activity in the subsequent recycling experiments.

To further explore the structure change of the catalyst during the reusability, the fresh and reused catalyst were investigated by XRD. As can be seen in Fig. $\mathrm{S} 3, \uparrow$ the characteristic peaks of sodium aluminate at $2 \theta=20.8,30.2,33.6,35.0,45.8,48.4,51.8$, 58.1, 61.1, 63.0 (JCPDS 00-019-1179 and 01-083-0316) were clearly observed in both fresh and reused catalyst, and the peak

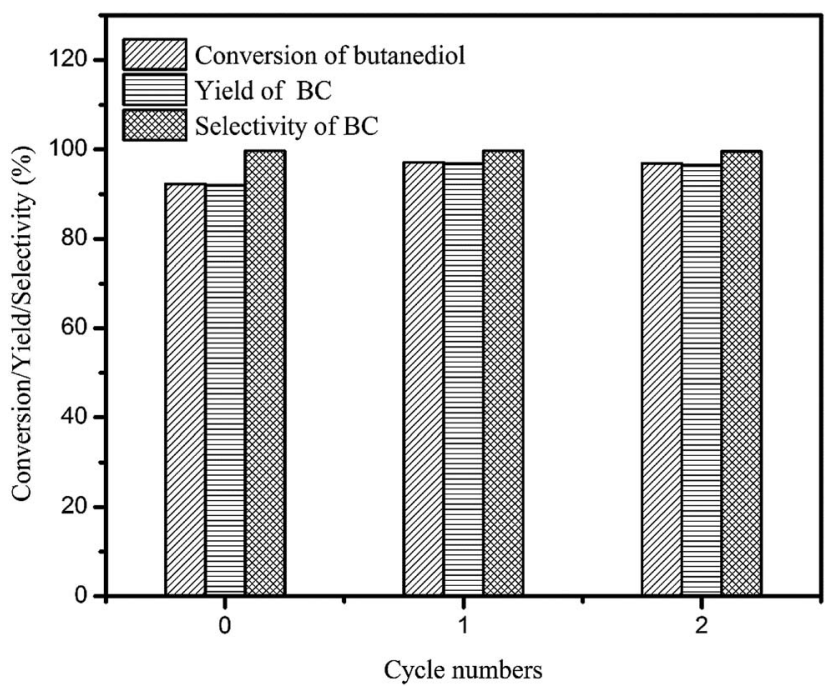

Fig. 5 Reuse of catalyst $\mathrm{NaAlO}_{2}$. (Reaction conditions: butanediol: 0.05 mol; $n(\mathrm{DMC}) / n$ (butanediol) = 4; catalyst: $10 \mathrm{wt} \%$ of $\mathrm{NaAlO}_{2}$ based on butanediol; temperature: $120{ }^{\circ} \mathrm{C}$; reaction time: $30 \mathrm{~min}$.)

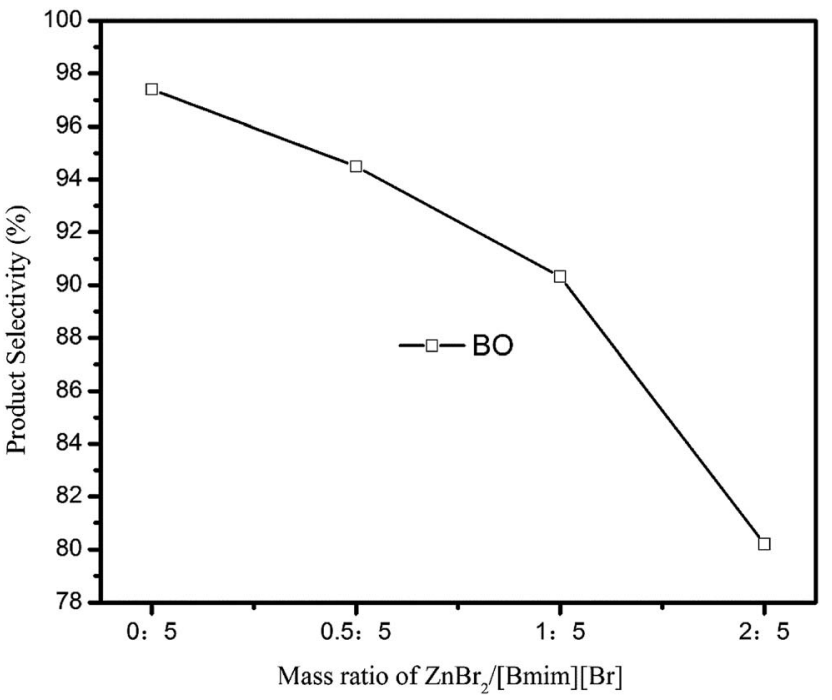

Fig. 6 Effect of promoter/catalyst mass ratio on $\mathrm{BO}$ synthesis. (Reaction conditions: BC: 40 g; catalyst: 0.83 g; reaction time: 90 min; vacuum degree: $0.005 \mathrm{MPa}$.)

strength is basically the same. These results suggested that crystalline structure and catalyst activity primarily remained during the recycle process, the results of which is consistent with the experimental data.

From the spectra in Fig. S4, $\uparrow$ we can see that the peaks at 3300 to $3700 \mathrm{~cm}^{-1}$ and $1650 \mathrm{~cm}^{-1}$ are attributed to the bending mode of the $-\mathrm{OH}$ group of physically adsorbed water and the peaks at $1455 \mathrm{~cm}^{-1}$ corresponding to the carbonate after it was exposed to the air. The peaks at $810,610,560$ and $490 \mathrm{~cm}^{-1}$ had no obvious changes after the sample was used for the reaction, indicating that the composition of $\mathrm{NaAlO}_{2}$ is stable under the reaction conditions used.

The basicity of the $\mathrm{NaAlO}_{2}$ (both before and after reaction) was evaluated by using $\mathrm{CO}_{2}$ temperature-programmed desorption (TPD) measurements as shown in Fig. S5. $\dagger$ The used

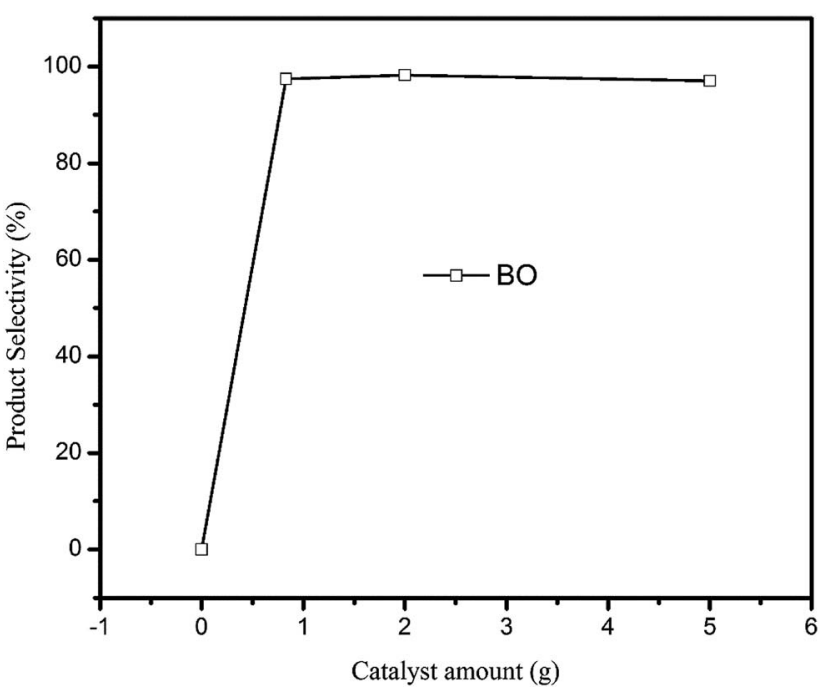

Fig. 7 Effect of catalyst amount on BO synthesis. (Reaction conditions: $\mathrm{BC}$ : $40 \mathrm{~g}$; reaction time: $90 \mathrm{~min}$; vacuum degree: $0.005 \mathrm{MPa}$.) 
$\mathrm{NaAlO}_{2}$ was separated by centrifugation and washed by ethanol. Then dried it at $100{ }^{\circ} \mathrm{C}$ for $4 \mathrm{~h}$. To evaluate the amount of different types of basic sites, the desorption curve can be arbitrarily differentiated into two regions corresponding to weak and strong basic sites (below or above $400{ }^{\circ} \mathrm{C}$, respectively). The desorption peaks at $256{ }^{\circ} \mathrm{C}$ for fresh $\mathrm{NaAlO}_{2}$ indicated basic sites of weak strength. The desorption peaks at $460{ }^{\circ} \mathrm{C}$ and $740{ }^{\circ} \mathrm{C}$ of used $\mathrm{NaAlO}_{2}$ can be attributed to the interaction of $\mathrm{CO}_{2}$ with strong basic sites because the higher temperature is needed to desorb the $\mathrm{CO}_{2}$ adsorbed on the more strongly basic sites.

The surface area is also tested using BET characterization. As shown in Table $\mathrm{S} 1, \dagger$ it can be observed that the BET surface area of the used $\mathrm{NaAlO}_{2}\left(13.6 \mathrm{~m}^{2} \mathrm{~g}^{-1}\right)$ was higher than that of fresh $\mathrm{NaAlO}_{2}\left(1.7 \mathrm{~m}^{2} \mathrm{~g}^{-1}\right)$. The enlarged surface area could be presumably attributed to the leaching of sodium element. Thus, more basic sites could be exposed.

\section{Synthesis of BO from decarboxylation of BC}

As aforementioned, imidazolium-based ionic liquids have been shown to be capable of catalyzing the decarboxylation of alkylene carbonates. In this work, $[\mathrm{Bmim}][\mathrm{Br}]$ ionic liquid was tested and applied as the catalyst for the decarboxylation of BC. With using ionic liquids $[\mathrm{Bmim}][\mathrm{Br}]$ as catalyst and $\mathrm{ZnBr}_{2}$ as promoter. The decarboxylation of $\mathrm{BC}$ can be effectively carried out, and the effect of reaction conditions, such as promoter/ catalyst mass ratio, catalyst amount, were investigated and discussed below.

Fig. 6 showed the influence of promoter/catalyst mass ratio on the $\mathrm{BO}$ synthesis with ionic liquids $[\mathrm{Bmim}][\mathrm{Br}]$ as catalyst and $\mathrm{ZnBr}_{2}$ as promoter. It can be observed that the selectivity of BO reduced from $97.4 \%$ to $80.2 \%$ with increasing the mass ratio from 0 to 2 . Therefore, the catalytic system without promoter is more superior choice in the selective synthesis of BO.

The effect of catalyst amount on the reaction was investigated, and the result is shown in Fig. 7. It is seen that the reaction could not occur without any catalyst. However, with the increase of ionic liquids $[\mathrm{Bmim}][\mathrm{Br}]$ from $0.83 \mathrm{~g}$ to $2.00 \mathrm{~g}$, the selectivity of BO slightly increased from $97.4 \%$ to $98.2 \%$. After that, the selectivity of $\mathrm{BO}$ reduced with the further increase of catalyst amount.

However, this result obtained is from the commercial $\mathrm{BC}$ rather than $\mathrm{BC}$ from the carbonylation of butanediol with DMC. Thus, in order to realize the two-step process, the synthesis of BO was furtherly carried out by using BC generated from butanediol. In this process, $\mathrm{BC}$ was firstly synthesized by the transesterification reaction of butanediol with DMC over $\mathrm{NaAlO}_{2}$ catalyst under the optimized reaction conditions. After the reaction, $\mathrm{BC}$ was separated from the product mixture and purified by vacuum distillation. The obtained $\mathrm{BC}$ was further used for the synthesis of $\mathrm{BO}$ over $[\mathrm{Bmim}][\mathrm{Br}]$ catalyst. As a result, $94.0 \%$ of separated $\mathrm{BO}$ yield could be obtained. In this sense, a novel approach for BO synthesis is realized by a twostep process of carbonylation and decarboxylation method for the first time.

The decarboxylation of butenyl carbonate was undertaken in the presence of several [Bmim]-based ionic liquids with different anions $\left(\mathrm{Cl}^{-}, \mathrm{I}^{-}, \mathrm{BF}_{4}{ }^{-}\right)$, and the effect of different IL on formation rate of the decarboxylation of butenyl carbonate was shown in Fig. S6. $\dagger$ It can be observed that IL with halide anions $[\mathrm{Bmim}][\mathrm{Cl}],[\mathrm{Bmim}][\mathrm{Br}]$ and $[\mathrm{Bmim}][\mathrm{I}]$ can catalyze the decarboxylation of $\mathrm{BC}$. The $\mathrm{FR}_{\mathrm{BO}}$ values are $8.7,3.9,1.2$, respectively, which are in the sequence of $[\mathrm{Bmim}][\mathrm{Cl}]>[\mathrm{Bmim}][\mathrm{Br}]>[\mathrm{Bmim}]$ $[\mathrm{I}]$. While the $[\mathrm{Bmim}]\left[\mathrm{BF}_{4}\right]$ can not catalyze the decarboxylation of BC. The selectivity of BO is $96.4 \%$ when the catalyst is [Bmim] [Cl], while the higher selectivity of $98.2 \%$ can be obtained when<smiles>CCC1COC(=O)O1</smiles>

$\mathrm{BC}$

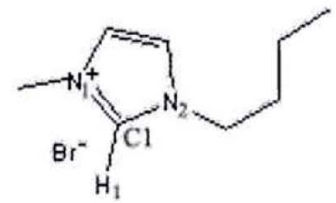

$[\mathrm{Bmim}][\mathrm{Br}]$
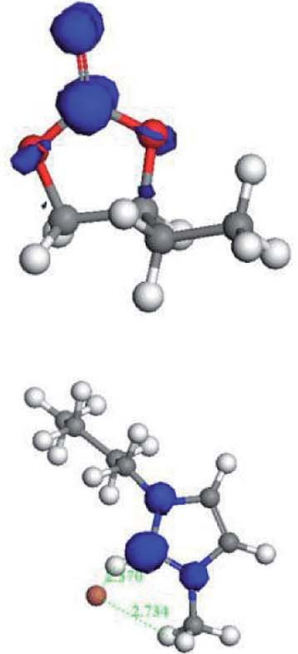

electropositivity attack

area
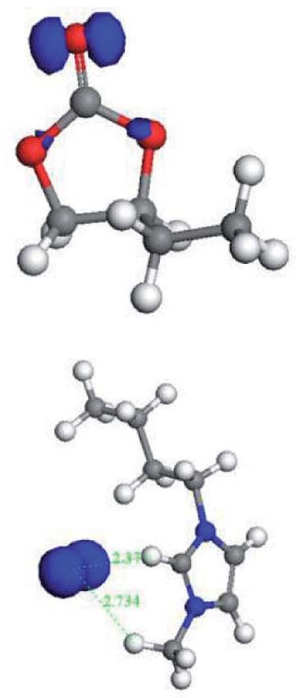

Nucleophilic attack

area

Fig. 8 Optimized structure of the $B C$ and the $[\mathrm{Bmim}][\mathrm{Br}]$. 


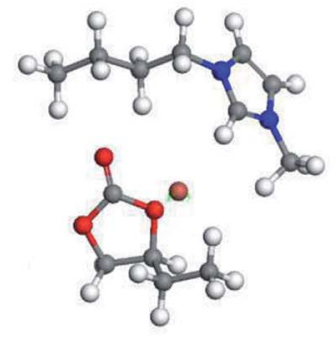

A

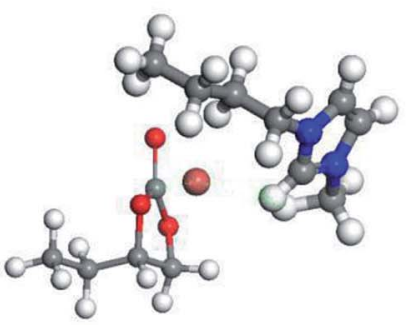

$\mathrm{D}$

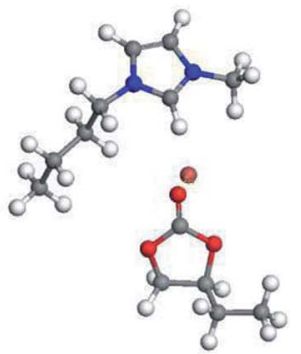

B

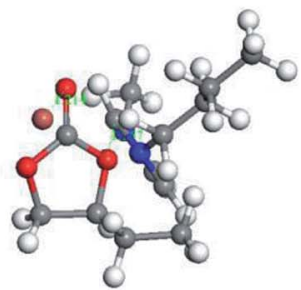

E

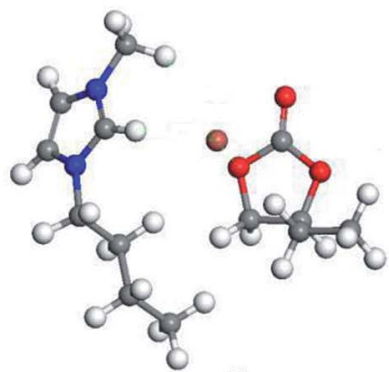

$\mathrm{C}$

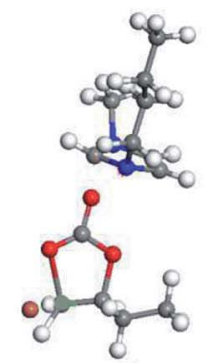

$\mathrm{F}$

Fig. 9 Six initial configurations in DFT calculation.

the catalyst is [Bmim] $[\mathrm{Br}]$. Thus, it is clear that anion of ILs have great impact on the decarboxylation of BC.

\section{Mechanism of the decarboxylation}

As it is shown in Fig. 7, the involvement of [Bmim] [Br] catalyst resulted in the $\mathrm{BO}$ product, while the yield of $\mathrm{BO}$ is negligible when there is no introduction of any catalyst. Thus, the DFT calculations were performed to reveal the interaction between $[\mathrm{Bmim}][\mathrm{Br}]$ and BC.

As showed in Fig. 8, it was found that the carbonyl oxygen $\mathrm{O} 1$ of the $\mathrm{BC}$ was the main nucleophilic attack area and the carbon $\mathrm{C} 1$ of the $\mathrm{BC}$ were the main electropositivity attack area. The nitrogen atoms of the $[\mathrm{Bmim}][\mathrm{Br}]$ were the main nucleophilic

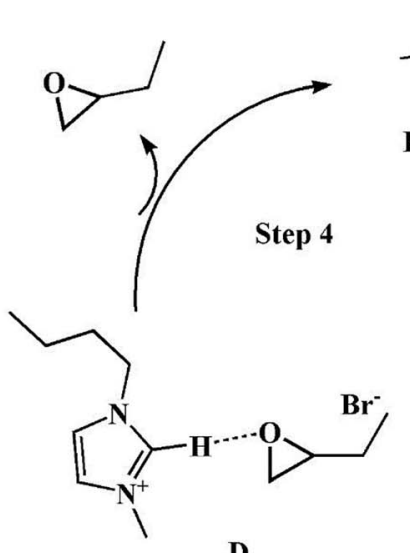

D

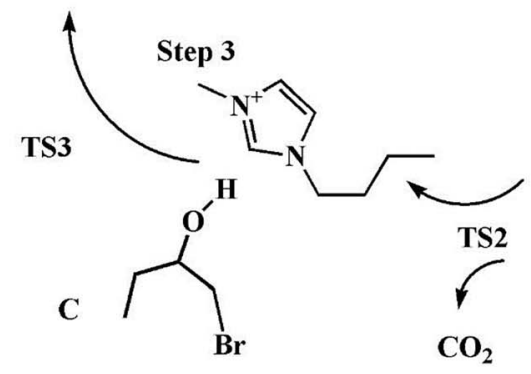<smiles>CCCCn1cc[n+](C)c1[Al]OC(=O)OC1OC(CC)C(CBr)O1</smiles>

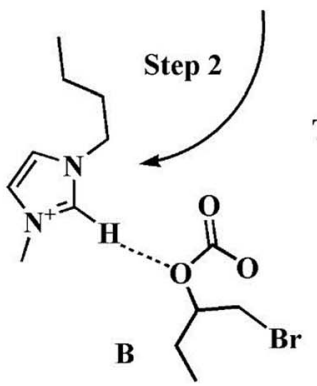

Scheme 2 The possible decarboxylation reaction mechanism. 


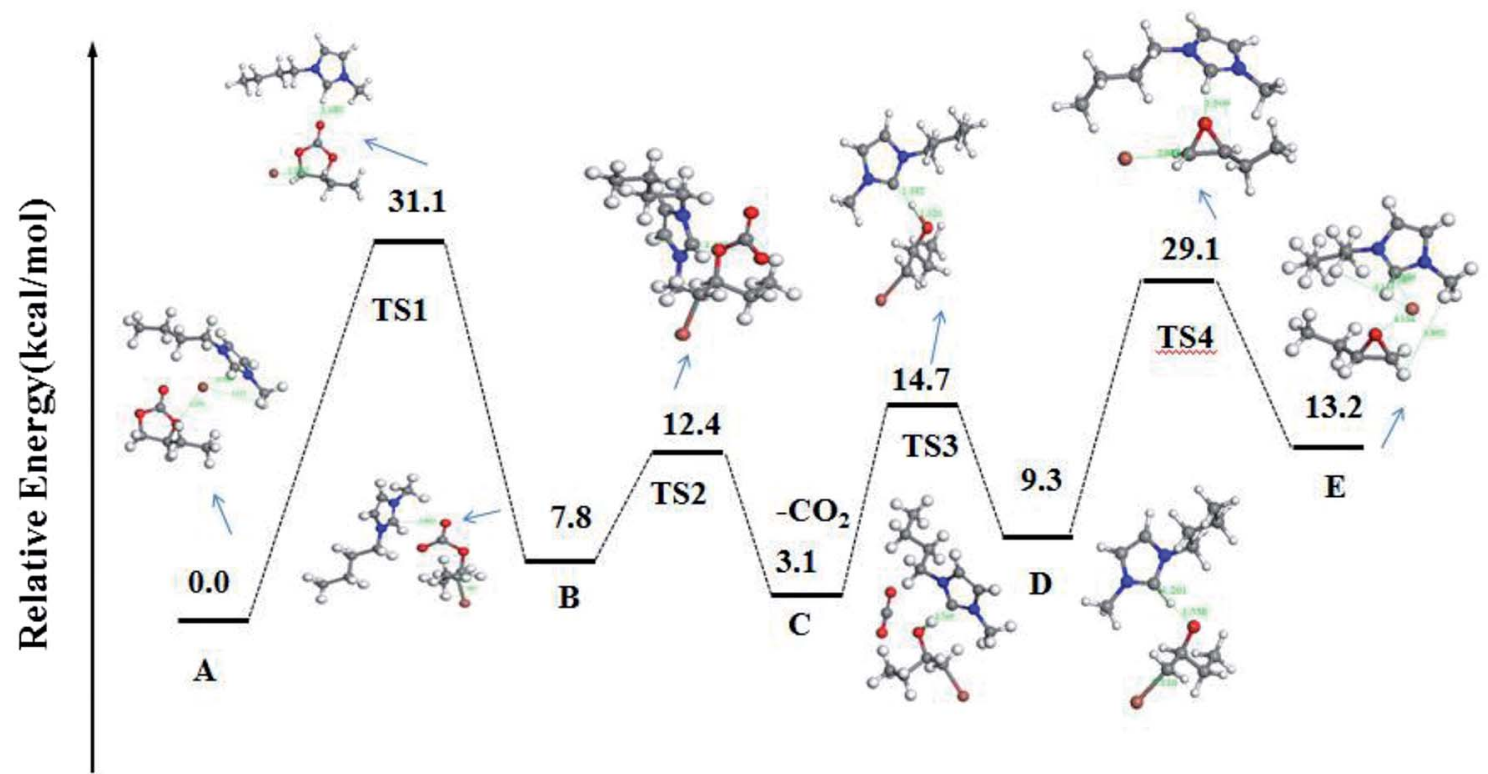

Fig. 10 Potential energy surface profiles of the [Bmim] [Br]-catalyzed process and the optimized geometries for the intermediates and transition states.

attack area. The $\mathrm{Br}^{-}$anions, which formed the hydrogen bonds of $\mathrm{C}-\mathrm{H} \cdots \mathrm{Br}$, was the main electropositivity attack area of $[\mathrm{Bmim}][\mathrm{Br}]$. Accordingly, six initial configurations between BC and $[\mathrm{Bmim}][\mathrm{Br}]$ was assumed and shown in Fig. 9.

According to the reports and DFT calculation results, a possible mechanism for decarboxylation was illustrated in Scheme $2 .{ }^{37,38}$ The optimized geometries for the intermediates and transition states were shown in Fig. $\mathrm{S} 7 \dagger$. The vibrational mode analysis shows that the reaction mechanism is reliable. When using vibrational mode analysis to elucidate the mechanism, we find that the only imaginary frequency of a transition state is always related to the breaking or forming bond. The potential energy surface profiles of the $[\mathrm{Bmim}][\mathrm{Br}]$-catalyzed process was shown in Fig. 10.

As shown in Scheme 2 and Fig. 10, first, the five-ring-opening step was the rate-limiting step with the highest energy barrier of $31.1 \mathrm{kcal} \mathrm{mol}^{-1}$. It was observed that the nucleophilic attack of $\mathrm{Br}$ anions on the least-hindered carbon $\mathrm{C} 2$ of $\mathrm{BC}$ played a very important role to break the covalent bond between the $\mathrm{C} 2$ and $\mathrm{O} 2$. Under the interaction with $\mathrm{Br}$ anions, $\mathrm{BC}$ was activated with the $\mathrm{C} 2-\mathrm{O} 2$ bond length enlarged from $1.45 \AA$ and $2.74 \AA$. However, a small probability was observed that the $\mathrm{C} 3$ of $\mathrm{BC}$ was nucleophilically attacked by $\mathrm{Br}$ anions because of the steric effect. Besides, the hydrogen bond between $\mathrm{H} 1$ and the carbonyl oxygen (O1) made the ring-opening much easier. Thus, the fivering-opening step made A convert into intermediate B via transition state TS1. Then, a new complex $\mathrm{C}$ was formed when $\mathrm{CO}_{2}$ was released into the reaction system via the TS2 with an energy barrier of $4.6 \mathrm{kcal} \mathrm{mol}^{-1}$. The third step is the proton transfer from $\mathrm{O} 3 \cdots \mathrm{H}$ to the nitrogen atom of the imidazolium ring along with the simultaneous loss of $\mathrm{Br}$ anion via transition state TS3 with an energy barrier of $11.4 \mathrm{kcal} \mathrm{mol}^{-1}$. Finally, the epoxy ring was obtained via an intramolecular nucleophilic attack, and the catalyst was regenerated (TS4) with an energy barrier of $29.1 \mathrm{kcal} \mathrm{mol}^{-1}$ (TS3). Besides, there was some negative effect of the hydrogen bonding between the $\cdot \mathrm{C} \cdots \mathrm{H}$ on the ring-closing event.

\section{Conclusions}

In conclusion, this paper firstly reported a novel approach for the synthesis of $\mathrm{BO}$ via decarboxylation of $\mathrm{BC}$ derived from butanediol. By using a cheap and easily available raw material $\mathrm{NaAlO}_{2}$ as catalyst, $99.7 \%$ BC selectivity and $96.2 \%$ BC yield can be obtained from the transesterification of butanediol with DMC, and the catalyst can be easily recovered and reused. Furthermore, BC can be effectively decarboxylated to $\mathrm{BO}$ when using $[\mathrm{Bmim}][\mathrm{Br}]$ as catalyst. Finally, $\mathrm{BO}$ was obtained by a twostep carbonylation and decarboxylation method for the first time by using butanediol as raw material. This new process is very significant for the production of epoxy compounds and the utilization of butanediol by-product inevitably generated in the process of coal to ethylene glycol.

\section{Conflicts of interest}

There are no conflicts of interest to declare.

\section{Acknowledgements}

"Transformational Technologies for Clean Energy and Demonstration", Strategic Priority Research Program of the Chinese Academy of Sciences, Grant No. XDA 21030600; National Natural Science Foundation of China (No. 21576272 and 21476244) and Youth Innovation Promotion Association CAS (2016046). 


\section{References}

1 Y. Zuo, M. Liu, M. T. Ma, C. S. Song and X. W. Guo, Ind. Eng. Chem. Res., 2017, 56, 7462-7467.

2 S. Sang, US Pat., US2018271191-A1, 2018.

3 J. Hilf, P. Schulze, J. Seiwert and H. Frey, Macromol. Rapid Commun., 2014, 35, 198-203.

4 I. Kim, J. T. Ahn, S. H. Chang, C. S. Yang and I. Park, Polymer, 2003, 44, 3417-3428.

5 L. I. Hua, W. Wang, R. Lin and X. She, CN Patent, CN101085763, 2010.

6 H. Fang, J. Ge, J. Li, Y. Xue and K. Zhang, CN Patent, CN104098531-B, 2016.

7 M. Hong, Y. Kim, H. Kim, H. J. Cho, M. H. Baik and Y. Kim, J. Org. Chem., 2018, 83, 9370-9380.

8 V. Onyenkeadi, S. Kellici and B. Saha, Energy, 2018, 165, 867876.

9 A. I. Adeleye, D. Patel, D. Niyogi and B. Saha, Ind. Eng. Chem. Res., 2014, 53, 18647-18657.

10 M. Alves, B. Grignard, R. Mereau, C. Jerome, T. Tassaing and C. Detrembleur, Catal. Sci. Technol., 2017, 7, 2651-2684.

11 W. L. Dai, W. Y. Yang, Y. Zhang, D. Wang, X. B. Luo and X. M. Tu, J. CO2 Util., 2017, 17, 256-262.

12 M. Ulusoy, E. Cetinkaya and B. Cetinkaya, Appl. Organomet. Chem., 2009, 23, 68-74.

13 R. H. Shi, W. W. Dong, Z. Han, T. Zhang and L. H. Zhang, Yingyong Huagong, 2013, 42, 2056-2060.

14 H. Li, W. Huang, X. Li and X. Gao, Ind. Eng. Chem. Res., 2016, 55, 9994-10003.

15 A. Yin, X. Guo, W.-L. Dai, H. Li and K. Fan, Appl. Catal., A, 2008, 349, 91-99.

16 S. Ramesh, F. Devred, V. D. B. Ludivine and D. P. Debecker, ChemCatChem, 2017, 10, 1398-1405.

17 Y. T. Algoufi, G. Kabir and B. H. Hameed, J. Taiwan Inst. Chem. Eng., 2016, 70, 179-187.

18 S. Wang, P. F. Hao, S. X. Li, A. L. Zhang, Y. Y. Guan and L. N. Zhang, Appl. Catal., A, 2017, 542, 174-181.

19 Y. K. Endah, S. K. Min, J. Choi, J. Jae, D. L. Sang and H. Lee, Catal. Today, 2016, 293, 136-141.

20 P. Zhang, L. Liu, M. Fan, Y. Dong and P. Jiang, RSC Adv., 2016, 6, 76223-76230.
21 V. P. Indran, N. A. S. Zuhaimi, M. A. Deraman, G. P. Maniam, M. M. Yusoff, T. Y. Y. Hin and M. H. A. Rahim, RSC Adv., 2014, 4, 25257-25267.

22 A. Axelsson, A. Antoine-Michard and H. Sunden, Green Chem., 2017, 19, 2477-2481.

23 T. Wan, P. Yu, S. Wang and Y. Luo, Energy Fuels, 2009, 23, 1089-1092.

24 R. Bai, P. Liu, J. Yang, C. Liu and Y. Gu, ACS Sustainable Chem. Eng., 2015, 3, 1292-1297.

25 M. M. Wang, M. A. A. Gasmalla, H. A. Tessema, X. Hua and R. J. Yang, Food Chem., 2017, 233, 151-158.

26 A. A. Marianou, C. M. Michailof, A. Pineda, E. F. Iliopoulou, K. S. Triantafyllidis and A. A. Lappas, ChemCatChem, 2016, 8, 1100-1110.

27 J. Kadokawa, Y. Iwasaki and H. Tagaya, Macromol. Rapid Commun., 2002, 23, 758-760.

28 R. Abdul-Karim, A. Hameed and M. I. Malik, RSC Adv., 2017, 7, 11786-11795.

29 J. S. Choi, F. S. H. Simanjuntak, J. Y. Oh, K. I. Lee, S. D. Lee, M. Cheong, H. S. Kim and H. Lee, J. Catal., 2013, 297, 248255.

30 S. M. Gade, M. K. Munshi, B. M. Chherawalla, V. H. Rane and A. A. Kelkar, Catal. Commun., 2012, 27, 184-188.

31 D. J. Darensbourg and A. D. Yeung, Green Chem., 2014, 16, 247-252.

32 X. L. N. Su, W. W. Lin, H. Y. Cheng, C. Zhang, Y. Wang, X. J. Yu, Z. J. Wu and F. Y. Zhao, Green Chem., 2017, 19, 1775-1781.

33 L. Zhao, P. He, L. Wang, M. Ammar, Y. Cao and H. Li, Catal. Today, 2016, 281, 392-401.

34 L. Wang, M. Ammar, P. He, Y. Li, Y. Cao, F. Li, X. Han and H. Li, Catal. Today, 2016, 281, 360-370.

35 S. Fischer and M. Karplus, Chem. Phys. Lett., 1992, 194, 252261.

36 Y. Gao, W. Peng, Z. Ning, W. Wei and Y. Sun, J. Mol. Catal. A: Chem., 2011, 351, 29-40.

37 X. Meng, H. Y. He, Y. Nie, X. Zhang, S. J. Zhang and J. Wang, ACS Sustainable Chem. Eng., 2017, 5, 3081-3086.

38 L. Wang, H. Li, S. Xin, P. He, Y. Cao, F. Li and X. Hou, Appl. Catal., A, 2014, 471, 19-27. 